\title{
Estado nutricional de idosos em grupos de convivência
}

\author{
Carmen Andréia Angst*, Cristiana Basso"*, Thiago Durand Mussoi"*; Tereza Cristina Blasi" ${ }^{* * *}$,

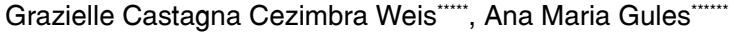

\section{Resumo}

A mudança do perfil nutricional, epidemiológico e demográfico da população idosa, implica adequações da estrutura social para atender as demandas na área da saúde e garantia de segurança alimentar. Esta pesquisa, de caráter transversal, descritivo e quantitativo, teve por objetivo avaliar o estado nutricional de idosos de dois grupos de convivência da cidade de Santa Maria, no estado do Rio Grande do Sul. Foram coletados dados antropométricos, peso e altura, dados dietéticos pelo recordatório 24h, e parâmetros bioquímicos da glicose e frações de colesterol. Participaram do estudo trinta indivíduos idosos, de ambos os sexos, em que classificados pelo índice de massa corpórea (IMC), 57\% tinham sobrepeso e $43 \%$ estavam eutróficos. De acordo com os parâmetros bioquímicos, apresentaram hipercolesterolemia e níveis baixos de HDL-C 56\% das mulheres com sobrepeso, e hiperglicemia $45 \%$ das muIheres eutróficas. Dos homens com sobrepeso, em $17 \%$ houve elevação do LDL-C, e em 33\% triglicerídeos e glicemia elevados. Conforme o recordatório $24 \mathrm{~h}$, houve consumo de $36,8 \%$ de lipídeos pelos homens obesos e $31,9 \%$ pelas mulheres obesas, e consumo insuficiente de fibras por $93 \%$ dos idosos. Dessa forma, os resultados referentes ao estado nutricional mostraram, a prevalência de obesidade, o perfil lipídico e glicêmico alterado em exames bioquímicos, o que pode estar relacionado à alimentação inadequada desses idosos.

Palavras-chave: Envelhecimento. Saúde. Hábitos alimentares. Obesidade.

* Nutricionista graduada pelo Centro Universitário Franciscano, Santa Maria (RS). Endereço para correspondência: Av. Nossa Senhora das Dores, 849, 97050-531, Santa Maria-RS. E-mail: ir.m.carmenandreia@ gmail.com

*** Nutricionista. Especialista em Nutrição Clínica. Mestre em Ciência e Tecnologia de Alimentos. Docente do curso de Nutrição do Centro Universitário Franciscano, Santa Maria (RS).E-mail: cristiana@unifra.br

**** Nutricionista. Especialista em Nutrição Clínica pela Associação Brasileira de Nutrição - Asbran. Mestre em Distúrbios da Comunicação Humana. Docente do curso de Nutrição do Centro Universitário Franciscano, Santa Maria (RS). E-mail: tdmussoi@gmail.com

***** Nutricionista. Especialista em Terapia Nutricional. Mestre em Ciência e Tecnologia de Alimentos. Docente do curso de Nutrição do Centro Universitário Franciscano, Santa Maria (RS).E-mail: tcb29@hotmail.com Nutricionista graduada pelo Centro Universitário Franciscano, Santa Maria (RS). E-mail: grazielle. castagna@gmail.com

******* Acadêmica de Nutrição e Farmácia no Centro Universitário Franciscano, Santa Maria (RS). E-mail: anamariagules@gmail.com

$\rightarrow$ http://dx.doi.org/10.5335/rbceh.v12i1.4615

Recebido em: 07/01/2015. Aceito em: 08/04/2015 


\section{Introdução}

Os seres humanos são chamados a cultivar e cuidar da vida, conferindo-lhe qualidade, dignidade e condições necessárias para o desdobramento natural e harmonioso de todas as habilidades que lhe são conferidas. Cuidar da saúde do idoso é missão e tarefa da sociedade, da comunidade e da família de cada indivíduo. O Estatuto do Idoso, instituído pela Lei $\mathrm{n}^{\mathrm{o}} 10.741$, de $1^{\circ}$ de outubro de 2003 , visa proteger o idoso garantindo-lhe direito à vida, à alimentação, à cultura, ao lazer, à cidadania, ao respeito e à dignidade, bem como possibilitar que os idosos possam usufruir de todas as oportunidades que preservem sua saúde física, mental, suas condições sociais, de liberdade e autonomia.

O envelhecimento populacional é determinado pela mudança da faixa etária das pessoas. Segundo o Ministério da Saúde (BRASIL, 2010), no Brasil, a definição de pessoa idosa aplica-se a indivíduos com idade de 60 anos ou mais. Considerado como um fenômeno natural, o envelhecimento avança de forma rápida, e de acordo com o Instituto Brasileiro de Geografia e Estatística (2010), o Censo Demográfico apontou 20,5 milhões de pessoas consideradas idosas, e, para 2025, prevê que cerca de 32 milhões de pessoas atinjam a idade de 60 anos ou mais.

O perfil demográfico apresentou, a partir de 1980, redução dos níveis de fecundidade e mortalidade, acelerando o processo de envelhecimento populacional. De acordo com o Ministério da Saúde (Brasil, 2010), em 2008, as crianças de
0 a 14 anos correspondiam a $26,47 \%$ da população total, e os idosos de 65 anos ou mais, a $6,7 \%$. Há uma projeção de que, em 2050, as crianças representarão $13,15 \%$, enquanto que os idosos ultrapassarão $22,7 \%$ da população total.

O aumento da expectativa de vida dos idosos, no contexto da transição epidemiológica, caracteriza-se pela mudança do perfil de morbidade e mortalidade. As doenças transmissíveis retrocederam, no entanto, prevalecem as doenças crônicas degenerativas não transmissíveis, como diabetes, hipertensão arterial, obesidade, acidente vascular encefálico, fraturas por quedas, insuficiência cardíaca, doença pulmonar obstrutiva crônica e demência, como Alzheimer. Essas situações implicam tratamentos médicos prolongados, internações, serviços de saúde, e cuidados especiais (BRASIL, 2010, 2014).

Em paralelo, ocorre a transição nutricional, com problemas alimentares e nutricionais, decorrentes da preferência por alimentos industrializados, como doces, refrigerantes e gorduras saturadas, bem como a oferta de alimentos pré-preparados, substituindo a aquisição de alimentos in natura de origem animal e vegetal, em quantidade e qualidade adequadas, que promovem aporte nutricional saudável (BRASIL, 2008, 2014; FREITAS; PHILIPPI; RIBEIRO, 2011).

O comportamento alimentar e o controle na ingestão de dietas balanceadas podem beneficiar a saúde do ser humano, dando suporte no combate de doenças crônicas degenerativas não transmissíveis. Segundo a V Diretriz Brasileira sobre Dislipidemias e Prevenção da Ateroscle- 
rose (2013) e as Diretrizes da Sociedade Brasileira de Diabetes (OLIVEIRA; VENCIO, 2014), o consumo aumentado de carboidratos, ácidos graxos saturados e trans, colesterol e quantidade excessiva de calorias acarreta elevação dos níveis séricos de colesterol e triglicerídeos, e tem interferência na regulação da glicemia.

A mudança do perfil nutricional, epidemiológico e demográfico implica adequações da estrutura social para atender as demandas na área da saúde e a garantia de segurança alimentar. Nesse contexto, as políticas de saúde têm como objetivo primordial contribuir para que os idosos cheguem a idades mais avançadas, com parâmetros de vida mais saudáveis, podendo desfrutar da velhice de forma ativa e consciente, dando sentido pleno à existência prolongada (BRASIL, 2014; SOCIEDADE BRASILEIRA DE GERIATRIAE GERONTOLOGIA, 2014).

Considera-se que a alimentação exerce papel fundamental na qualidade de vida do idoso, repercutindo em seu estado nutricional e de saúde global. Assim sendo, o presente estudo tem o objetivo de avaliar o estado nutricional de idosos em dois grupos de convivência, da cidade de Santa Maria, no Rio Grande do Sul.

\section{Materiais e Métodos}

Esta pesquisa, de caráter transversal, descritivo e quantitativo (PEREIRA, 2002), foi realizada com idosos participantes de dois grupos de convivência de terceira idade da cidade de Santa Maria-RS, no período de julho a dezembro de 2014. Foram incluídos no estudo os indivíduos maiores de 60 anos, sexo masculino e feminino, que estavam se alimentando por via oral, lúcidos e que aceitaram participar da pesquisa. Como critérios de exclusão, considerou-se a avaliação dos indivíduos em relação a doenças neurodegenerativas, pela aplicação do Mini-Exame do Estado Mental (Folstein; Folstein; Mchugh, 1975; BERTOLUCCI et al., 1994; BRUCKY et al., 2003), e ausência em alguma etapa da pesquisa.

A avaliação antropométrica foi realizada pela aferição de peso $(\mathrm{kg})$ e altura (m), de acordo com as recomendações do Ministério da Saúde (BRASIL, 2008). O peso corporal foi avaliado por meio de balança digital Filizola ${ }^{\circledR}$, sendo os indivíduos pesados descalços e com o mínimo de vestimentas possíveis. Para aferição da altura, foi utilizado estadiômetro portátil Sanny ${ }^{\circledR}$.

A classificação do estado nutricional, pelo Índice de Massa Corpórea (IMC), foi calculada pela razão entre o peso e a altura ao quadrado do indivíduo (IMC = peso/altura ${ }^{2}$ ), e classificado conforme Sisvan (BRASIL, 2008), em baixo peso $\leq 22 \mathrm{~kg} / \mathrm{m}^{2}$; eutrofia, de 22 a $27 \mathrm{~kg} / \mathrm{m}^{2}$; e sobrepeso, $\geq 27 \mathrm{~kg} / \mathrm{m}^{2}$, obtendo-se, assim, o estado nutricional do idoso.

Para a verificação do consumo alimentar, foi aplicado um teste piloto com a intenção de selecionar o melhor método para esse estudo. O teste foi realizado com oito idosos, utilizando o recordatório $24 \mathrm{~h}$ e o registro alimentar de três dias não consecutivos, incluindo um dia do final de semana, sábado ou domingo (FISBERG; MARCHIONI; COLUCCI, 2009; MUSSOI, 2014). Optou-se por utilizar o método recordatório $24 \mathrm{~h}$, 
considerando a melhor aceitabilidade por parte dos idosos abordados, sendo esse aplicado em diferentes dias da semana, como segunda-feira, quarta-feira e quinta-feira, com abordagem individual ao idoso.

Os dados referentes ao consumo alimentar foram coletados e analisados no programa dietWin Personal ${ }^{\circledR}$ (2011), para que se pudesse obter os valores de calorias, macronutrientes e fibras. A análise da adequação dos nutrientes efetuou-se conforme os valores recomendados pelas Dietary Reference Intakes (DRIs) (IOM, 2001, 2011), considerando o sexo e a faixa etária. Os macronutrientes foram avaliados pelos intervalos de distribuição aceitável dos macronutrientes (AMDR), a saber: carboidratos $45 \%$ a $65 \%$, proteínas $10 \%$ a $35 \%$ e lipídios $25 \%$ a $35 \%$. A média da necessidade estimada de energia (EER) foi obtida a partir de cada um dos membros dos grupos, pela equação proposta pelo Institute of Medicine (2001, 2011), que considera o estágio de vida, o gênero, o peso, a altura, o coeficiente de atividade física e o estado nutricional do indivíduo. $\mathrm{O}$ coeficiente de atividade física foi identificado pelos relatos dos idosos em relação a sua rotina diária.

Visando à investigação do perfil lipídico e glicêmico, as coletas sanguíneas dos idosos foram realizadas nos grupos de convivência, por profissionais da área da saúde, sob orientação e supervisão de um professor do curso de Biomedicina do Centro Universitário Franciscano, seguindo todos os cuidados de biossegurança. Foram coletados $10 \mathrm{ml}$ de sangue periférico de cada paciente. Os tubos foram encaminhados ao Laboratório Es- cola de Análises Clínicas (LEAC) dentro de uma caixa isotérmica $\left(2^{\circ} \mathrm{C}\right.$ a $\left.8^{\circ} \mathrm{C}\right)$, e imediatamente os tubos sem anticoagulante foram centrifugados por dez minutos a $3000 \mathrm{rpm}$. As análises bioquímicas para o perfil lipídico realizaram-se pelo método enzimático para determinação quantitativa em soro. A leitura foi feita em equipamento semiautomatizado Bioplus $^{\circledR}$, e os kits para as dosagens foram da marca Labtest ${ }^{\circledast}$. Todas as avaliações bioquímicas tiveram uma amostra controle (Qualitrol 1H). Para as análises hematológicas foram usados tubos com EDTA, e as amostras analisadas no equipamento ADVIA $60^{\circledR}$ (equipamento semiautomatizado).

A avaliação e a classificação do perfil lipídico seguiram parâmetros da V Diretriz Brasileira sobre Dislipidemias e Prevenção da Aterosclerose, da Sociedade Brasileira de Cardiologia (2013), e os níveis de glicose pela American Diabetes Association (ADA) (2013).

Os indivíduos que participaram da coleta de dados, referente à dieta alimentar e bioquímica, tiveram retorno dos resultados de maneira individual, com orientações nutricionais e esclarecimentos quanto aos hábitos alimentares.

A efetuação desta pesquisa deu-se após ser avaliada e aprovada pelo Comitê de Ética e Pesquisa do Centro Universitário Franciscano, conforme parecer número 705.492. O termo de consentimento livre e esclarecido foi apresentado aos integrantes, contendo as informações necessárias sobre as atividades e esclarecendo que a participação era de livre e espontânea vontade, certificando anonimato dos dados. 
Para a análise e tabulação dos dados, utilizaram-se os programas Microsoft Office Word $2007^{\circledR}$ e Microsoft Office Excel $2007^{\circledR}$, realizou-se, então, estatística descritiva obtendo-se resultados em média, desvio padrão e porcentagem.

\section{Resultados}

O presente estudo coletou e analisou dados de participantes de dois grupos de convivência da terceira idade, totalizando 46 idosos, dos quais participaram $65 \%$ $(\mathrm{n}=30)$, sendo $27 \%$ do sexo masculino $(n=8)$ e $73 \%$ do feminino $(n=22)$, que estavam de acordo com os critérios de inclusão. Foram excluídos 19\% $(n=9)$ dos indivíduos que ignoraram alguma das etapas da pesquisa, como coleta de sangue para efetuação dos exames bioquímicos, preenchimento do recordatório $24 \mathrm{~h}$ e aferição das medidas antropométricas, e $16 \%(n=7)$ se recusaram a participar da pesquisa.

A Tabela 1 apresenta a classificação do estado nutricional dos idosos estudados, que variou entre eutrofia e sobrepeso.

Tabela1 - Classificação do estado nutricional, consumo alimentar e necessidade calórica dos idosos de grupos de convivência. Santa Maria-RS, 2014

\begin{tabular}{l|r|r|l|r|r}
\hline \multicolumn{1}{c|}{$\begin{array}{c}\text { Gênero } \\
\mathrm{N}\end{array}$} & $\begin{array}{c}\text { Amostra } \\
\%(\mathrm{n})\end{array}$ & \multicolumn{1}{|c|}{ IMC } & Classificação & $\begin{array}{c}\text { Consumo } \\
\text { alimentar } \\
(\mathrm{m} \pm \mathrm{dp})\end{array}$ & $\begin{array}{c}\text { Necessidade } \\
\text { Calórica (DRIs) } \\
(\mathrm{m} \pm \mathrm{dp})\end{array}$ \\
\hline $\begin{array}{l}\text { Mulheres } \\
22\end{array}$ & $50(11)$ & $25 \pm 1,11$ & Eutrofia & $1428 \pm 551,42$ & $1812 \pm 119,13$ \\
\hline Homens & $25(11)$ & $29,97 \pm 2,24$ & Sobrepeso & $1429 \pm 388,93$ & $1900 \pm 123,17$ \\
\hline 8 & $75(6)$ & $31,47 \pm 5,54$ & Sobrepeso & $1749 \pm 653,12$ & $2445 \pm 243,72$ \\
\hline $\begin{array}{l}\text { Total } \\
30\end{array}$ & $43(13)$ & $25,09 \pm 1,38$ & Eutrofia & $1439 \pm 527,44$ & $1865 \pm 176,09$ \\
\hline
\end{tabular}

Fonte: elaboração dos autores.

Nota: * Dietary Reference Intakes (IOM, 2001, 2011)

Para a investigação do consumo alimentar dos idosos, foi utilizado o método recordatório $24 \mathrm{~h}$. Sendo que, ao realizar o teste piloto, constatou-se que $75 \%$ dos idosos demonstraram certa resistência em preencher o registro alimentar, fazendo alegações como: "Terei dificuldade em anotar"; "Isto não vou conseguir fazer"; "Costumo comer a mesma coisa quase todos os dias". O recordatório alimentar $24 \mathrm{~h}$ aplicado foi respondido por $100 \%$ dos idosos sem dificuldades, pelo fato de terem hábito alimentar contínuo, conforme seus relatos. 
Tabela 2 - Consumo alimentar de macronutrientes e fibras dietéticas por idosos de dois grupos de convivência. Santa Maria-RS, 2014

\begin{tabular}{|c|c|c|c|c|c|}
\hline Variável & \multicolumn{2}{|c|}{ Eutrofia } & \multicolumn{2}{|c|}{ Sobrepeso } & \multirow{2}{*}{$\begin{array}{c}\text { Total } \\
n=30 \\
\text { (m } \pm d p)\end{array}$} \\
\hline Macronutrientes & $\begin{array}{l}\text { Mulheres } \\
\qquad \mathrm{n}=11 \\
(\mathrm{~m} \pm \mathrm{dp})\end{array}$ & 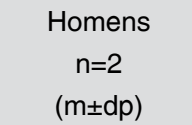 & $\begin{array}{l}\text { Mulheres } \\
\qquad \mathrm{n}=11 \\
(\mathrm{~m} \pm \mathrm{dp})\end{array}$ & $\begin{array}{l}\text { Homens } \\
\qquad \mathrm{n}=6 \\
(\mathrm{~m} \pm \mathrm{dp})\end{array}$ & \\
\hline Carboidratos(\%) & $51,55 \pm 9,90$ & $57,5 \pm 9,19$ & $50,9 \pm 19,09$ & $42,67 \pm 8,50$ & $49,63 \pm 10,11$ \\
\hline Proteínas(\%) & $18,55 \pm 1,41$ & $14 \pm 1,41$ & $17,1 \pm 4,24$ & $20,00 \pm 4,82$ & $17,93 \pm 4,24$ \\
\hline Lipídios (\%) & $30,00 \pm 8,49$ & $28,5 \pm 10,61$ & $31,9 \pm 24,04$ & $36,8 \pm 13,11$ & $32,03 \pm 10,83$ \\
\hline Colesterol (mg) & $305,20 \pm 311,28$ & $87,30 \pm 102,66$ & $207,9 \pm 188$ & $297 \pm 148$ & $252 \pm 229$ \\
\hline Gerdures catured & $17,66 \pm 10,33$ & $13,63 \pm 9,63$ & $20 \pm 12,71$ & $25,41 \pm 12,93$ & $19,66 \pm 11,63$ \\
\hline Gorauras saturadas (g) & ${ }^{*} 11 \%$ & $8,2 \%$ & $13 \%$ & $13 \%$ & $9 \%$ \\
\hline $\begin{array}{l}\text { Gorduras mono } \\
\text { insaturadas(g) }\end{array}$ & $13,25 \pm 8,70$ & $16,54 \pm 5,37$ & $13,3 \pm 9,06$ & $24,21 \pm 12,78$ & $15,69 \pm 10,15$ \\
\hline Gordurac noli_incaturadac & $17,87 \pm 31,04$ & $16,52 \pm 14,32$ & $19 \pm 31,16$ & $16,77 \pm 15,11$ & $18,02 \pm 26,73$ \\
\hline Gorauras poll-Insaturadas & ${ }^{\star}{ }^{*} 11 \%$ & $9 \%$ & $11 \%$ & $8 \%$ & $8 \%$ \\
\hline Gorduras trans & $0,31 \pm 0,34$ & $0,17 \pm 0,06$ & $0,37 \pm 0,33$ & $0,46 \pm 0,53$ & $0,35 \pm 0,36$ \\
\hline Fibras (g) & $18,92 \pm 11,0$ & $31,2 \pm 14,2$ & $14,68 \pm 4,8$ & $14,82 \pm 8,86$ & $17,37 \pm 9,03$ \\
\hline
\end{tabular}

Fonte: elaboração dos autores com base na I Diretriz sobre o consumo de Gorduras e Saúde Cardiovascular (Santos et al. 2013).

Nota: * Porcentagem em relação ao VET (referência < 7\% do VET); ** Porcentagem em relação ao VET (referência até $10 \%$ do VET)

Ao realizar a avaliação do estado nutricional do idoso, torna-se importante a utilização dos parâmetros bioquímicos para monitorar e identificar possíveis carências nutricionais e riscos para doen- ças crônicas degenerativas que possam comprometer a saúde do idoso (MUSSOI, 2014). A Tabela 3 demonstra o perfil lipídico e glicêmico dos idosos. 
Tabela 3 - Parâmetros bioquímicos dos idosos de dois grupos de convivência. Santa Maria-RS, 2014

\begin{tabular}{|c|c|c|c|c|c|}
\hline \multirow{3}{*}{ Variável } & \multirow{3}{*}{ Referência } & \multicolumn{2}{|c|}{ Eutrofia } & \multicolumn{2}{|c|}{ Sobrepeso } \\
\hline & & $\begin{array}{c}\text { Mulheres } \\
n=11\end{array}$ & $\begin{array}{c}\text { Homens } \\
\mathrm{n}=2\end{array}$ & $\begin{array}{c}\text { Mulheres } \\
n=11\end{array}$ & $\begin{array}{c}\text { Homens } \\
\mathrm{n}=6\end{array}$ \\
\hline & & $\%(n)$ & $\%(n)$ & $\%(n)$ & $\%(n)$ \\
\hline \multicolumn{6}{|l|}{${ }^{*}$ Colesterol total $(\mathrm{mg} / \mathrm{L})$} \\
\hline Desejável & $<200$ & $55(6)$ & - & $19(2)$ & $50(3)$ \\
\hline Limítrofe & $200-239$ & $35(4)$ & $50(1)$ & $35(4)$ & $33(2)$ \\
\hline Alto & $\geq 240$ & $9(1)$ & $50(1)$ & $46(5)$ & $17(1)$ \\
\hline \multicolumn{6}{|l|}{${ }^{*} \mathrm{LDL}(\mathrm{mg} / \mathrm{L})$} \\
\hline Ótimo & $<100$ & $19(2)$ & - & - & $33(2)$ \\
\hline Desejável & $100-129$ & $27(3)$ & - & $27(3)$ & $17(1)$ \\
\hline Limítrofe & $130-159$ & $27(3)$ & $50(1)$ & $19(2)$ & $33(2)$ \\
\hline Alto & $160-189$ & $27(3)$ & - & $56(6)$ & - \\
\hline Muito alto & $\geq 190$ & - & $50(1)$ & - & $17(1)$ \\
\hline \multicolumn{6}{|l|}{${ }^{*} \mathrm{HDL}(\mathrm{mg} / \mathrm{L})$} \\
\hline Desejável & $>60$ & - & - & $19(2)$ & $17(1)$ \\
\hline Limítrofe & $60-40$ & $73(8)$ & $50(1)$ & $72(8)$ & $83(5)$ \\
\hline Baixo & $<40$ & $27(3)$ & $50(1)$ & $9(1)$ & - \\
\hline \multicolumn{6}{|l|}{ *Triglicerídios(mg/L) } \\
\hline Desejável & $<150$ & $73(8)$ & $100(2)$ & $81(9)$ & $67(4)$ \\
\hline Limítrofe & $150-200$ & $19(2)$ & - & $19(2)$ & $33(2)$ \\
\hline Alto & $200-449$ & $9(1)$ & - & - & - \\
\hline Muito alto & $\geq 500$ & - & - & - & - \\
\hline \multicolumn{6}{|l|}{${ }^{\star \star *}$ Glicemia (mg/L) } \\
\hline Desejável & $<100 \mathrm{mg} / \mathrm{dl}$ & $56(6)$ & $100(2)$ & $81(9)$ & $67(4)$ \\
\hline Tolerância diminuída & $>100 a<126$ & $36(4)$ & - & $19(2)$ & $33(2)$ \\
\hline Diabetes mellitus & $\geq 126$ & $9(1)$ & - & - & - \\
\hline
\end{tabular}

Fonte: elaboração dos autores.

Nota: *V Diretriz Brasileira sobre Dislipidemia e Prevenção da Aterosclerose do Departamento de Aterosclerose da Sociedade Brasileira de Cardiologia, 2013; ${ }^{* *}$ ADA. Novas Diretrizes de Diabetes Mellitus. American Diabetes Association, 2013. 
Na Tabela 4, verifica-se que $56 \%$ das mulheres com sobrepeso, pelo índice de massa corporal, apresentam hipercolesterolemia, bem como níveis de HDL-C abaixo dos níveis recomendados. Outro parâmetro importante é a hiperglicemia em $45 \%$ das mulheres idosas eutróficas, apresentando igualmente níveis elevados de colesterol e triglicerídeos.

Tabela 4 - Parâmetros bioquímicos relacionados a dislipidemias, segundo classificação IMC dos idosos de dois grupos de convivência. Santa Maria-RS, 2014

\begin{tabular}{l|r|r|r|r|r}
\hline \multirow{2}{*}{ Variável } & \multicolumn{2}{|c|}{ Eutrofia } & \multicolumn{2}{c|}{ Sobrepeso } & \multicolumn{2}{c}{ Total } \\
\cline { 2 - 6 } & $\begin{array}{c}\text { Mulheres } \\
\mathrm{n}=11\end{array}$ & $\begin{array}{c}\text { Homens } \\
\mathrm{n}=2\end{array}$ & $\begin{array}{c}\text { Mulheres } \\
\mathrm{n}=11\end{array}$ & $\begin{array}{c}\text { Homens } \\
\mathrm{n}=6\end{array}$ & $\mathrm{n}=30$ \\
\cline { 2 - 6 } & $\%(\mathrm{n})$ & $\%(\mathrm{n})$ & $\%(\mathrm{n})$ & $\%(\mathrm{n})$ & $\%(\mathrm{n})$ \\
\hline *Hipercolesterolemia & $27(3)$ & $50(1)$ & $56(6)$ & $17(1)$ & $36(11)$ \\
*Hipertrigliceridemia & $28(3)$ & - & $19(2)$ & $33(2)$ & $23(7)$ \\
*Hiperlipidemia & $9(1)$ & - & $9(1)$ & - & $6(2)$ \\
*HDL-C baixo & $64(7)$ & $50(1)$ & $64(7)$ & - & $50(15)$ \\
**Hiperglicemia & $45(5)$ & & $19(2)$ & $33(2)$ & $30(9)$ \\
\hline
\end{tabular}

Fonte: elaboração dos autores.

Nota: *V Diretriz Brasileira sobre Dislipidemia e Prevenção da Aterosclerose do Departamento de Aterosclerose da Sociedade Brasileira de Cardiologia, 2013; ${ }^{*}$ ADA, Novas Diretrizes de Diabetes Mellitus. American Diabetes Association, 2013.

\section{Discussões}

O perfil nutricional dos idosos tem se modificado, e isso pode estar associado ao consumo alimentar, à obtenção e ao preparo dos alimentos. Constata-se que o idoso tem menor gasto energético, diminuição da massa muscular e aumento do tecido adiposo (MAURICIO; FEBRONE; GAGLIARDO, 2014).

Conforme resultados obtidos na pesquisa de Garcia, Godoy e Pereira (2012), com cinquenta idosos participantes de um projeto social, os dados antropométricos mostraram $45 \%$ de sobrepeso, assim como risco aumentado para o desenvolvimento de doenças cardiovasculares.
Outro estudo foi realizado por Venturini et al. (2013), com base polulacional pelo Instituto de Geriatria e Gerontologia da Pontifícia Universidade Católica do Rio Grande do Sul (PUCRS), em parceria com a Prefeitura Municipal de Porto Alegre. O trabalho abrangeu 304 idosos, 217 mulheres e 87 homens, com idade de 60 a maiores de 80 anos, dentre eles, houve sobrepeso em $73,8 \%$ $(n=160)$ das mulheres e em 67,8\% ( $n=59)$ dos homens. A epidemia da obesidade vem crescendo no decorrer dos anos, o que pode ser verificado em muitos estudos, que identificam causas similares, relacionadas a estilo de vida, fatores 
genéticos e sociais, além de distúrbios nutricionais (BRASIL, 2010).

Segundo dados do IBGE (2010), o excesso de peso acomete $50,1 \%$ dos homens e $48 \%$ das mulheres. O Ministério da Saúde (Brasil, 2014) aponta como fatores contribuintes dessa problemática o padrão alimentar e o gasto energético diminuído.

A ingestão alimentar média das mulheres foi de $1428,74 \mathrm{kcal}$ (desvio padrão $\pm 460,94$ ), e dos homens, em média, de 1685,39 kcal (desvio padrão $\pm 603,13$ ), considerando que o método aplicado pode conter falhas como déficit de memória do entrevistado e falta de precisão nas medidas caseiras utilizadas (FISBERG; MARCHIONI; COLUCCI, 2009; MUSSOI, 2014). A estimativa para a necessidade de energia para as mulheres foi de 1856,16 kcal (desvio padrão $\pm 126,48$ ) e para os homens $2372,34 \mathrm{kcal}$ (desvio padrão $\pm 245,64$ ), constatando-se, dessa forma, que o consumo alimentar dos idosos é inferior à necessidade média de energia estimada pelas DRIs. Outro estudo, realizado por Carreira (2013), com treze idosos, mostrou consumo alimentar médio de 1178,55 kcal (desvio padrão $\pm 227,39)$ para as mulheres, e 1564,42 kcal (desvio padrão $\pm 396,16$ ), para os homens.

A ingestão alimentar diária do idoso tende a diminuir com a perda gradativa do apetite e aspectos sensoriais do paladar e olfato, ocorrendo a redução da sensibilidade para gostos primários como salgado e doce. As condições de saúde bucal também ficam prejudicadas pelo uso de próteses e pela falta de arcada dentária, que podem levar a dificuldades de mastigação (BRITO; PAVARINI, 2012).

No presente estudo, $83 \%(n=25)$ dos idosos relataram uso de próteses dentárias. Ocorre, ainda, a diminuição da mobilidade, da prática de atividade física, possíveis complicações metabólicas e situações familiares, em que o idoso muitas vezes acaba fazendo as refeições sozinho, e por isso sente-se desmotivado, e até mesmo depressivo.

Um aspecto peculiar que também foi mencionado, diz respeito ao comportamento alimentar, pois os idosos afirmaram consumir regularmente as mesmas porções e tipos de alimentos, priorizando o que cada estação oferece em gêneros alimentícios, fato esse já constatado na aplicação do teste piloto. Conforme Philippi e Aquino (2009), observou-se que o idoso, com o passar dos anos, adquire hábitos alimentares que são efetuados de forma sistemática, desde o despertar, pela manhã, até o findar do dia. Segundo relatos, costumam usar os mesmos talheres, copos e xícaras, fazer compras sempre no mesmo lugar, e priorizam horários estabelecidos para a realização das refeições.

A alimentação adequada exerce papel fundamental para o envelhecimento saudável, e leva o idoso a vivenciar de maneira positiva sua condição fisiológica, ambiental, social e nutricional, promovendo melhora na qualidade de vida e nas atividades diárias (MAURICIO; FEBRONE; GAGLIARDO, 2014).

Os idosos, quando analisados de maneira global, conforme Tabela 2, mostraram consumo alimentar adequado de macronutrientes, mas quantidade insu- 
ficiente de fibras. No entanto, o perfil alimentar lipídico apresenta alterações na qualidade em relação à ingestão de colesterol e gorduras saturadas. $\mathrm{Na}$ análise segundo os gêneros, os homens que apresentaram sobrepeso tiveram consumo excessivo de lipídios, 36,8\% (desvio padrão $\pm 13,11$ ), e houve consumo elevado de lipídios pelas mulheres com sobrepeso, de $31,9 \%$ (desvio padrão $\pm 24,04)$.

$\mathrm{Na}$ ingestão dietética das fibras, que colaboram na regulação dos níveis de glicose e colesterol, identificou-se diferenças no consumo entre idosos com sobrepeso e eutrofia. Segundo os resultados obtidos, somente dois homens eutróficos $(25 \%)$ alcançaram a recomendação de fibras, consumindo $31,2 \mathrm{~g}$ (desvio padrão $\pm 14,2$ ) por dia. A Sociedade Brasileira de Diabetes (OLIVEIRA; VENCIO, 2014), preconiza no mínimo o consumo de $20 \mathrm{~g}$ por dia de fibras, e o Institute of Medicine (2001), 21g para mulheres e $30 \mathrm{~g}$ para homens.

Os idosos tendem a escolher alimentos mais macios, fáceis de mastigar, dando preferência ao consumo de frutas em forma de sucos ou cozidas, legumes cozidos, e pão branco. Os alimentos integrais vem sendo introduzidos de forma gradativa na alimentação dos idosos.

De acordo com o Ministério da Saúde (BRASIL, 2014), evidencia-se o consumo elevado de alimentos ricos em açúcar, gorduras saturadas, trans e sal, assim como consumo inadequado de carboidratros complexos e fibras. Outro parâmetro importante, de acordo com dados do IBGE (2011), é que houve estabilização de consumo de frutas e hortaliças, que representam menos da metade da recomendação, e redução de alimentos como ovos, peixes, leguminosas, raízes, tubérculos e arroz.

Os alimentos mais consumidos pelos dois grupos de idosos foram café, arroz, carne, feijão, leite integral, pão francês, banana, maçã, laranja, tomate, alface e biscoito salgado. Esses alimentos vão ao encontro do consumo identificado pela pesquisa realizada com 100 idosos da Zona Leste de São Paulo, no ano de 2008, em que a prevalência de alimentos consumidos foram café, arroz branco, feijão, açúcar refinado, leite integral, adoçante líquido, pão francês, leite desnatado, alface e margarina (FREITAS; PHILIPPI; RIBEIRO, 2011). Segundo o Inquérito Nacional de Alimentação (2008-2009), analisado por faixas etárias, os idosos tiveram consumo prevalente de café, arroz, feijão, pão francês, carne bovina, óleos e gorduras, sucos e refrescos, banana, biscoito salgado e leite integral (IBGE, 2011).

A população da Região Sul do Brasil, de acordo com Venturini et al. ( 2013), tem hábito de consumir níveis elevados de proteína animal, como carne de gado, frango, porco, linguiça, toucinho, e ressalta, ainda, que os homens tem margem maior de ingestão e, em decorrência disso, apresentam obesidade. Junto à fonte proteica da carne, consomem-se, simultaneamente, os lipídios compostos basicamente de gordura saturada, que contém os ácidos graxos de cadeia longa, aumentando os níveis de LDL. $\mathrm{O}$ alto consumo de carne, leite integral e derivados, pão francês e demais carboidratos pobres em fibras, pode estar 
correlacionado com os resultados obtidos no presente estudo, referentes à hipercolesterolemia, hipertrigliceridemia e baixos níveis de HDL-C.

O aumento dos níveis de colesterol pode estar relacionado à ingestão dietética, mas deve-se levar em consideração que o envelhecimento traz alterações metabólicas no processo da lipólise, ocorrendo maior liberação de ácidos graxos e glicerol. A hiperlipidemia constituise do excesso de lipídios circulantes na corrente sanguínea (V DIRETRIZ BRASILEIRA DE DISLIPIDEMIAS E PREVENÇÃO DA ATEROSCLEROSE, 2013).

O tecido adiposo tem a reserva do colesterol, e essa aumenta com a idade, influenciando no peso corpóreo. Quando acontece a substituição de ácidos graxos saturados por ácidos graxos monoinsaturados, há redução do LDL-C. Planos alimentares com carboidratos elevados, maiores que $50 \%$, elevam os triglicerídeos e diminuem o HDL-C, aumentam a glicemia e a insulinemia. Os tipos de carboidratos, como açúcar branco de adição, refrigerantes, biscoitos doces, su$\cos$, bolos e sorvetes, estão diretamente ligados aos níveis de lipídeos (SANTOS et al., 2013; OLIVEIRA; VENCIO, 2014).

O estudo realizado em Porto AlegreRS, abrangendo 304 idosos, mostrou alterações laboratoriais nos homens idosos com obesidade, em que $56 \%$ apresentaram HDL-C baixo e 50\% hipertrigliceridemia. Das mulheres obesas, $70 \%$ apresentaram hipercolesterolemia, $67 \%$ de HDL-C baixo, e 64\% hipertrigliceridemia. Esses resultados foram atribuídos ao sedentarismo dos idosos, ao elevado consumo de alimentos ricos em açúcar e gordura (VENTURINi et al., 2013).

$\mathrm{Na}$ pesquisa de Garcia, Godoy e Pereira (2012), com 50 idosos do sexo masculino e feminino, observou-se glicemia $>100 \mathrm{mg} / \mathrm{l}$ em $65 \%$ dos idosos, $45 \%$ com triglicerídeos $\geq 200 \mathrm{mg} / \mathrm{l}$, e $25 \%$ com colesterol total elevado $\geq 240 \mathrm{mg} / \mathrm{l}$. Entretanto, foram encontrados dados favoráveis relacionados à alimentação equilibrada e à prática de atividade física.

Pela análise do consumo alimentar dos dois grupos de idosos do presente estudo, constatou-se possíveis fatores que podem estar correlacionados com os parâmetros bioquímicos. No consumo alimentar de 46\% ( $n=5)$ das idosas eutróficas, verificou-se ingestão excessiva de carboidratos no almoço, com elevado índice glicêmico, como por exemplo arroz e batata, arroz e massa, sendo que $35 \%$ $(\mathrm{n}=4)$ apresentaram tolerância diminuída a glicose e níveis baixos de HDL-C, o que vai ao encontro de resoluções das Diretrizes da Sociedade Brasileira de Diabetes (OLIVEIRA; VENCIO, 2014).

Nas idosas com sobrepeso observouse níveis de LDL-C elevado em 56\% $(n=6)$, podendo atribuir-se essa condição ao consumo de alimentos ricos em gordura como salsichão, lombo recheado, linguiça de porco, ovo frito, margarina, cerveja, torradas, biscoito salgado, maionese, docinhos, tortas. Outro fator a ser considerado é a prática do jantar semelhante ao almoço, em porções menores, abrangendo $64 \%(\mathrm{n}=7)$ das idosas, enquanto que $36 \%(n=4)$ costumam ingerir refeição semelhante ao desjejum. 
Aspecto esse observado igualmente nos idosos homens com sobrepeso, visto que $67 \%(n=4)$ consomem jantar semelhante ao almoço e $33 \%(\mathrm{n}=2)$ costumam ingerir café com leite e pão, semelhante ao desjejum. Nos dados bioquímicos ocorreu elevação do LDL-C em 17\% ( $n=1)$, triglicerídeos em $33 \%(n=2)$, fato esse podendo ser atribuído ao consumo de carne de porco, maionese, refrigerante e açúcar.

Em relação às mulheres que apresentaram eutrofia, constatou-se que somente $6,6 \%(\mathrm{n}=2)$ tinham a prática do jantar semelhante ao almoço, sendo que 93,4 \% ( $\mathrm{n}=9$ ) costumam ingerir refeição semelhante ao desjejum. Os homens eutróficos relataram consumir jantar semelhante ao almoço, contudo, percebeu-se que a alimentação diária desses estava adequada quanto à quantidade de fibras dietéticas, que auxiliam no metabolismo e na absorção dos nutrientes.

Conforme dados coletados, os idosos mostraram consumo elevado de alimentos ricos em gordura, que de certa forma são saborosos e macios. No entanto, o consumo elevado de leite integral, margarina e carne de gado e porco, tortas e maionese, pode interferir nos parâmetros bioquímicos. Quanto à ingestão alimentar dos carboidratos, como arroz branco, massa, pão francês e batata inglesa, é válido encorajar os idosos a ingerir alimentos que contenham fibras, como arroz e pão integral, farelo de aveia, que melhoram seu perfil lipídico e glicêmico. Aconselha-se restringir a ingestão elevada de carboidratos na mesma refeição, como arroz com batata e arroz com massa. Essas condutas po- dem auxiliar no controle da glicemia e na regulação dos níveis de triglicerídeos.

Recomenda-se consumo diário de frutas, verduras e legumes, por serem alimentos que oferecem nutrientes e fibras importantes no combate de morbidades relacionadas ao envelhecimento, como dislipidemias e doenças crônicas degenerativas. Diante dos dados encontrados neste estudo, torna-se evidente que os hábitos alimentares adequados contribuem de forma efetiva na saúde do idoso, conferindo-lhe qualidade de vida no envelhecimento. Por isso a necessidade de orientação alimentar por um profissional nutricionista com o objetivo de adequar a alimentação dos idosos é evidente.

Segundo relatos dos idosos, eles praticam atividades físicas leves, como realização de tarefas domésticas, participação do grupo em atividades semanais, que oferecem dança, ginástica, jogo de carta, viagens, passeios, ações cumunitárias e palestras, tudo incentivando um estilo de vida saudável. O fato de os idosos integrarem um grupo de convivência traz efeitos benéficos à saúde deles, proporcionando oportunidades de maior conhecimento e orientação, novos laços de amizade e experiências, evitando a acomodação diária.

Segundo Fazzio (2012) é importante estimular as interações sociais dos idosos, o convívio com pessoas queridas, parentes, com a finalidade de promover autoestima e independência em relação às suas atividades diárias e rotina alimentar. Considera-se que a alimentação possui papel fundamental na qualidade de vida do idoso, repercutindo em seu estado nutricional e na saúde global. 
Todos os idosos dos grupos de convivência participantes desta pesquia receberam retorno individualizado dos resultados da avaliação nutricional, com entrega dos exames bioquímicos realizados e orientações conforme a necessidade de cada um, como para perda de peso, regulação da glicemia e redução do colesterol LDL, triglicerídeos, elevação do HDL-C, e orientações para uma alimentação saudável.

Podem ser considerados fatores limitantes da pesquisa as questões vinculadas à memória de alimentos que os idosos tenham ingerido e as quantidades, embora esses tenham rotina alimentar sistemática. O recordatório $24 \mathrm{~h}$ realizado nas segundas-feiras com alguns dos participantes pode ter interferido na obtenção dos dados, por retratar a refeição do final de semana. Outro aspecto relaciona-se quanto à persistência dos idosos em participar de todas as etapas da pesquisa, e até mesmo certa insegurança em integrar o grupo de pesquisa.

\section{Conclusão}

Ao realizar o presente estudo, pela avaliação nutricional dos idosos de dois grupos de convivência, conclui-se que o envelhecimento é determinado por um longo percurso e, com o passar dos anos, forma-se um alicerce em relação à alimentação, costumes, gostos peculiares, horários, preferências e intolerâncias. Os resultados referentes ao estado nutricional, com prevalência de obesidade, perfil lipídico e glicêmico alterados, pelos exames bioquímicos, podem estar relacionados à alimentação inadequada desses idosos.
Houve boa aceitação das orientações nutricionais, após os resultados dos exames bioquímicos. O idoso como excelência da maturidade humana comunica suas potencialidades, experiências, fragilidades e estilo de vida. Deles se pode aprender a construir formas e maneiras eficientes para contribuir com o cuidado da saúde, de modo evidenciado, da nutrição. Acompanhar seu ritmo alimentar, viabilizar acompanhamento nutricional, promover a dinâmica do envelhecimento com qualidade de vida, fazem parte desse processo. Torna-se pertinente orientar os idosos de maneira clara sobre a alimentação saudável, bem como monitorar suas necessidades físicas, psicológicas, sociais e espirituais.

\section{Nutritional state of elderly in social centers}

\section{Abstract}

Changes in nutritional, epidemiological and demographic profiles in the elderly population imply adaptations in the social structure to comply with the demands in the area of health and food safety guarantee. This transversal, descriptive and quantitative research aimed at assessing the nutritional conditions of elderly in social centers, in the city of Santa Maria, RS. Anthropometric data, weight and height, dietetic data through a 24-hour report and biochemical patterns of glycose and cholesterol fractions data were collected. Thirty subjects participated in the study, of both genders, classified by their body mass index (BMI), from which $57 \%$ of them were overweight and $43 \%$ were eutrophic. According to biochemical parameters, $56 \%$ of the overweight women presented hypercholesterolemia and low levels of HDL-C 
while $45 \%$ of the eutrophic women presented hyperglycemia. There was an elevation of LDL-C in $17 \%$ of overweight men, and $33 \%$ of them had elevated triglycerides and glycemia. According to the 24-hour report, there was an intake of $36,8 \%$ of lipid by obese men and $31,9 \%$ by obese women and an insufficient intake of fibers by $93 \%$ of the elderly. Thus, the results concerning nutritional state, prevailing obesity, and lipidic and glycemic profiles altered by biochemical exams may be related to those elderly's inadequate feeding.

Keywords: Aging. Health. Feeding Habits. Obesity.

\section{Referências}

AMERICAN DIABETES ASSOCIATION. Diagnosis and classification of diabetes mellitus. Diabetes Care, American Diabetes Association Supl 1, v. 36, p. 11-66, 2013.

BERTOLUCCI P. H. F. et al. O Mini-Exame do Estado Mental em uma população geral: impacto da escolaridade. Arquivos de Neuro-Psiquiatria, São Paulo, v. 52, n. 1, p. 1-7, 1994.

BRASIL. Ministério da Saúde. Atenção à saúde da pessoa idosa e envelhecimento. Brasília, DF, dez. 2010. Biblioteca Virtual em Saúde do Ministério da Saúde. Disponível em: <http:// www.saude.gov.br/bvs>. Acesso em: dez. 2014.

Ministério da Saúde. Estatuto do Idoso. Brasília: Ministério da Saúde, 2003. Disponível em: <http://www.saude.gov.br/ bvs >. Acesso em: nov. 2014.

. Ministério da Saúde. Secretaria de Vigilância à Saúde. Secretaria de Atenção à Saúde. Diretrizes e recomendações para o cuidado integral de doenças crônicas não-transmissíveis: promoção da saúde, vigilância, prevenção e assistência. Brasília: Ministério da Saúde, 2008. Disponível em: <http://www.saude.gov.br/bvs>. Acesso em: dez. 2014.
Ministério da Saúde. Vigilância alimentar e nutricional - SISVAN: orientações básicas para a coleta, o procedimento a análise de dados e a informação e serviços de saúde. Brasília, DF, 2008. Disponível em: $<$ http://www.saude.gov.br/bvs > . Acesso em: out. 2014.

Ministério da Saúde. Secretaria de Atenção à Saúde. Departamento de Atenção Básica. Estratégias para o cuidado da pessoa com doença crônica: obesidade. Brasília: Ministério da Saúde, 2014.Disponível em: <http://www.saude.gov.br/bvs>. Acesso em: dez. 2014.

BRITO, T. R. P.; PAVARINI, S. C. I. Relação entre apoio social e capacidade funcional de idosos com alterações cognitivas. Revista Latino-Americana Enfermagem, São Paulo, v. 20 , n. 4 , p. $3-8,2012$.

BRUCKI S. M. D. et al. Sugestões para o uso do Mini-Exame do Estado Mental no Brasil. Arquivos de Neuro-Psiquiatria, São Paulo, v. 63, n. 3, p. 777-781B, 2003.

CARREIRA, T. V. Relação entre ingestão alimentar e a composição corporal em idosos institucionalizados. 2013. 60f. Dissertação (Mestrado em Nutrição Clínica) - Cooperativa de Ensino Superior, CRL, Instituto Superior de Ciências da Saúde Egas Moniz, Repositório Científico de acesso aberto em Portugal, 2013.

FAZZIO, D. M. G. Envelhecimento e qualidade de vida - uma abordagem nutricional e alimentar. Revista de Divulgação Científica Sena Aires, Goiás, v. 1, n. 1, jan.-jun. 2012.

FISBERG, R. M.; MARCHIONI, D. M. L.; COLUCCI, A. C. A. Avaliação do Consumo Alimentar e da Ingestão de Nutrientes na Prática Clínica. Arquivos Brasileiros de Endocrinologia e Metabologia, São Paulo, v. 53, n. 5, p. 617-624, 2009.

FOLSTEIN, M. F.; FOLSTEIN, S. E.; MCHUGH, P. R. Mini-Mental State: a practical method for grading the cognitive state of patients for clinician. Journal of Psychiatric 
Research,Department of Psychiatry, University of Oregon Medical School, Portland, Oregon 97201, U.S.A., v. 12, n. 3, p. 189-198, 1975.

FREITAS, A. M. P.; PHILIPPI, S. T.; RIBEIRO, S. M. L. Listas de alimentos relacionadas ao consumo alimentar de um grupo de idosos: análises e perspectivas. Revista Brasileira de Epidemiologia, São Paulo, v. 14, n. 1, p. 161-177, 2011.

GARCIA, P. P. C.; GODOY, S. L.; PEREIRA, T. A. Saúde nutricional de idosos. Aspectos bioquímicos, antropométricos e alimentares. Ensaios e Ciência: Ciências Biológicas, Agrárias e da Saúde, Campo Grande, v. 16, n. 4, p. 25-37, 2012.

IBGE. INSTITUTO BRASILEIRO DE GEOGRAFIA E ESTATÍSTICA. Censo Demográfico de 2010: Distribuição da população por sexo, segundo os grupos de idade. 2010.

IBGE. INSTITUTO BRASILEIRO DE GEOGRAFIA E ESTATÍSTICA. Pesquisa de Orçamentos Familiares 2008-2009: tabela de medidas referidas para os alimentos consumidos no Brasil. Rio de Janeiro: IBGE, 2011.

INSTITUTE OF MEDICINE. Food and Nutrition Board. Dietary Reference Intakes for Energy, Carboydrates, Fiber, Fat, Fatty Acids, Cholesterol, Proteins, and Aminoacids (macronutrients). Washington, DC: National Academy Press, 2001.

INSTITUTE OF MEDICINE. Food and Nutrition Board (US). Dietary Reference Intakes for Calcium and Vitamin D. Washington, DC: National Academy Press; 2011.

MAURICIO, L. S.; FEBRONE, R. R.; GAGLIARDO, L. C. Avaliação da frequência de consumo alimentar de idosos participantes do projeto integrar. Revista Rede de cuidados em Saúde, Rio de Janeiro, v. 8, n. 1, p. 1-14, 2014.

MUSSOI, T. D. Avaliação Nutricional na prática clínica: da gestação ao envelhecimento. Rio de Janeiro: Guanabara Koogan, 2014.
OLIVEIRA, José Egidio Paulo de; VENCIO, Sérgio (Org.). Diretrizes da Sociedade Brasileira de Diabetes: 2013-2014/Sociedade Brasileira de Diabetes. São Paulo: AC Farmacêutica, 2014. p. 1-360.

PHILIPPI, S. T.; AQUINO, R. C. Nutrição Clínica Estudos de casos comentados. Barueri, São Paulo: Manole, 2009.

PEREIRA, G. M. Epidemiologia: teoria e prática. Rio de Janeiro: Guanabara Koogan, 2002.

SANTOS R. D. et al. Sociedade Brasileira de Cardiologia. I Diretriz sobre o consumo de Gorduras e Saúde Cardiovascular. Arquivo Brasileiro de Cardiologia, Rio de Janeiro, v. 100, n. 1, Suplemento 3, p. 1-40, jan. 2013.

SOCIEDADE BRASILEIRA DE CARDIOLOGIA. V Diretriz Brasileira sobre Dislipidemia e Prevenção da Aterosclerose do Departamento de Aterosclerose da Sociedade Brasileira de Cardiologia. Arquivos Brasileiros de Cardiologia, Rio de Janeiro, v. 101, n. 4, Suplemento 1, p. 1-22, out. 2013.

SOCIEDADE BRASILEIRA DE GERIATRIA E GERONTOLOGIA. Carta Aberta À População Brasileira. Disponível em: <http://sbgg. org.br/envelhecimento-no-brasil-e-saude-do-idoso-sbgg-divulga-carta-aberta-a-populacao-2/>. Acesso em: $4 \mathrm{dez} .2014$

VENTURINI, C. D. et al. Prevalência de obesidade associada à ingestão calórica, glicêmica e perfil lipídico em uma amostra populacional de idosos do Sul do Brasil. Revista de Geriatria e Gerontologia, Rio de Janeiro, v. 16, n. 3, p. 591-601, 2013.

THE NUTRITION SCREENING INITIATIVE. Incorporating Nutrition Screening and Interventions into Medical Practice. A Monograph for Physicians. Washington D.C. US: American Academy of Family Physicians; The American Dietetic Association; National Council on Aging Inc., 1994. 